\title{
Towards Analytical Modeling for Persuasive Design Choices in Mobile Apps
}

\author{
Hamid Mukhtar \\ National University of Sciences \& Technology (NUST) \\ 44000, Islamabad, Pakistan
}

\begin{abstract}
Persuasive technology has emerged as a new field of research in the past decade with its applications in various domains including web-designing, human-computer interaction, healthcare systems, and social networks. Although persuasive technology has its roots in psychology and cognitive sciences, researchers from the computing disciplines are also increasingly interested in it. Unfortunately, the existing theories, models, and frameworks for persuasive system design fall short due to absence of systematic design processes mostly used in the computing domains as well as lack of support for appropriate post-analysis.

This work provides some insight into such limitations and identifies the importance of analytical modeling for persuasion in mobile applications design. The authors illustrate, using a case study, that appropriate mathematical models can be applied together with user modeling to develop a persuasive system that will allow the designer to consider several design choices simultaneously.
\end{abstract}

Keywords-goal; intent; analytics; modeling; feedback

\section{INTRODUCTION}

Persuasive technology has its roots in psychology and human behavior and much of the early work done was focused on using websites and mobile phones [1][2][3] as persuasion media; however, as the technology matured and people had increased access to sophisticated devices and systems, it became more natural to design appropriate interactive interfaces and specialized tools as persuasive media. Researchers developed various methodologies [4], models [5] and architectures [6] that could serve as guidelines for designing a wide range of persuasive systems irrespective of the persuasion medium.

Due to such research efforts, persuasive technology has matured in the recent years and it has emerged as a separate field of research. Notably it has found its place in various applications of Human-Computer Interaction (HCI). In fact, persuasive design has recently got much attention and significant role in designing products varying from hand-held devices and household items to software products including websites and mobile applications. However, it was not until very recently when people realized that integrating "analytics" can leverage the persuasion power significantly. That's why, there have been efforts in integrating data logging and analysis in different systems designed with the objective of persuading the users [7][8]. Based upon these trends, the authors present here our view on the need for analytical models in persuasion.

\section{A. The Need for Analytical Models}

Fogg's Behavior Model (FBM) [9] defines behavior as a product of three factors: motivation, ability and triggers, and their subcomponents. While in theory this model serves as a good guide for understanding human behavior - and for identifying the target component to work on for inducing the desired behavior - it does not specify a mathematical or computational model or any hint on how to integrate it into such an existing model. This creates a major hindrance in designing persuasive systems that can be evaluated on the basis of expert or machine analysis. Most of the persuasive systems in the literature report about achieving significant change in the behavior of subjects; however, they do not specify to what extent a given component of the FBM was successful on a particular subject. In fact, such analysis cannot be made possible without appropriate analytical models. What if we can design an analytical model of a persuasive system that can not only provide qualitative but also quantitative analysis of the persuasion goal?

At the core of the analytical model, we can have a mathematical or computational model appropriate for each application scenario where persuasion is needed. Having a separate mathematical model for each persuasive situation provides the ability to fine tune the various characteristics associated with persuasion. It also implies that the designer has the ability to adopt the persuasion strategies to individuals or classes of individuals rather than applying the same strategies indiscriminately to all users.

This paper approaches the domain of persuasive technology from data analytics point-of-view with two main objectives. First, the authors want to emphasize that by integrating analytical models as part of the persuasive design leverages the persuasion process and will lead to enhanced outcomes. Second, the authors want to present an analytical model and, subsequently, describe how we can represent a certain human behavior as a mathematical model for persuasion. The author will also describe how this basic model allows persuasion to be personalized to each person's target behavior based upon their past performance.

This article presents the preliminary work on the importance of analytics in persuasion and is based upon the learning from the previous work on the design and development of persuasive healthcare applications [10][11][12]. Nevertheless, it also draws on the experiences of the work done by others in the domain of persuasive technology and pointers to them are provided throughout the article.

The rest of this article is organized as following. Section II provides the background and overview of the related work. Section III identifies some persuasive design elements which 
form the basis for the definition of the analytical model presented in Section IV. Section V describes a case study developed using the analytical model. Section VI concludes this article with some future directions.

\section{BACKGROUND AND RELATED WORK}

The various studies related to Behavior Change Support Systems (BCSS) [13][14] and particularly the Persuasive System Design (PSD)[5] process provide some model for designing persuasive systems. However, while such model may provide a strong theoretical background to understand the broad spectrum of persuasion, they are not sufficiently detailed to help a designer think in more concrete terms. This has resulted in a large gap between the theory and practice of persuasive technology.

For example, most of the work in persuasive technology start with a focus on Fogg's Behavior Model [9] and designing persuasive strategies for increasing one or more of its ingredients, namely, motivation, ability, or trigger. However, this is where the theory disappears and the focus is turned towards design elements of persuasion related to user interaction and perception. This is evident from the numerous research efforts such as [2][3][7][15]. In contrast, the author believes that by considering some analytical model, it becomes more instructive to consider various additional factors that are outlined below.

Fogg's behavior grid [16] identifies 35 ways a behavior can be changed or induced. The behavior grid categorizes the behavior on the basis of two dimensions: type of behavior change and time or schedule. Accordingly, a target behavior may fall into one of these 35 categories represented by a cell in the grid. Although the behavior grid does not restrict that a given behavior may fall into one category only, it also does not give any clue on mapping a target behavior on more than one cells over an extended period of time. Due to the absence of a formal mechanism, such mappings cannot be done systematically.

Fogg and Hreha [17] simplified the behavior grid to 15 states. The behavior axis has three types: dot (one-time), span (for a set duration) and path(permanent change). Each of these behaviors has five flavors: green, blue, purple, grey and black characterizing if a behavior is familiar or not and whether the intention is to start, increase, decrease or stop the behavior. Of these 15 behavior targets, the designer will spot one and persuade the user for that particular behavior duration and flavor. This will be in one of the 15 cells of the grid where the desired behavior duration and flavor intersect.

Although the current research does not concern the behavior grid, the interest here is in defining models that may place a user into one of the cell of the grid based on certain criteria. Then the user can be moved from cell to cell as his/her behavior is refined. During this process, certain elements from the area of gamification are borrowed, such as the notion of levels or progress. The objective is to justify the fact that by integrating certain level of analytics one can enhance the persuasion process and greatly help the designer identify a number of relevant persuasion strategies. Also, by integrating the concepts of context and user preferences from the lessons learned in the domain of ubiquitous computing, persuasion can be made more meaningful to the user.

\section{Designing for Analytics in Persuasion}

Recently, business intelligence and machine learning have gained much attention due to increased importance and capabilities of analytics in many fields. Thus, recently many persuasive technologies have been designed on top of analytics [2][8][18]. But in the absence of some analytical model, such efforts are not reusable in different contexts or for different problems. Based upon our previous experiences of developing persuasive systems of different nature, some common design factors have been identified and combined into a unified analytical model for persuasion.

In the remaining part of this section, these factors are identified and then in the next section the model is described. The following scenario is adapted for the purpose of explanation.

1) Example Scenario: Consider the problem of environmental hazards or climate effects resulting from excessive usage of electricity which is mostly generated from natural sources such as coal, petroleum or nuclear energy; all of them are exhaustive in nature. To minimize the environmental impacts due to electricity usage, a non-governmental body decides to persuade people to reduce electricity consumption and to move towards renewable source of energy such as solar energy. Thus, the design will include to deal with two behaviors either simultaneously or independently one of them. Keeping the persuasion goal in mind, we now describe how a persuasion designer can design some viable solution for inducing the desired target behavior in users.

\section{A. Difference between Persuader and User Goals}

Clearly, the intent of the persuader is to protect the environment and one possible way is by reducing the electricity consumption. Having the clearly defined goal of persuader, the designer may design persuasive strategies related to the Grey Path cell in the Fogg's behavior grid: decreasing a behavior that is always performed. For example, the designer may be tempted to start by persuading the consumers that reducing electricity consumption may be a noble cause of saving the humanity. A persuasion strategy would be informing the users about these negative effects so that they can make informed decisions about reducing electricity consumption.

Although this is a direct translation of the persuader's intent into a persuasion objective, it cannot be said for sure if it can be mapped to most of the users' intent. In other words, the persuasion strategy reduce usage to save environment may represent the persuader's intent but not necessary the user's intent. Also, the desired behavior may not be just about reducing a particular habit but alternative behaviors should also be considered.

For a persuasion strategy to be successful, the designer has to come up with a strategy or set of strategies that match user's goal. The challenge here lies in finding the user intent and then doing a translation of the persuader's intent into the user's intent. For example, the stated persuasion strategy of saving environment can be redefined as reduce electricity usage to save money. This strategy can be then put into practice by 


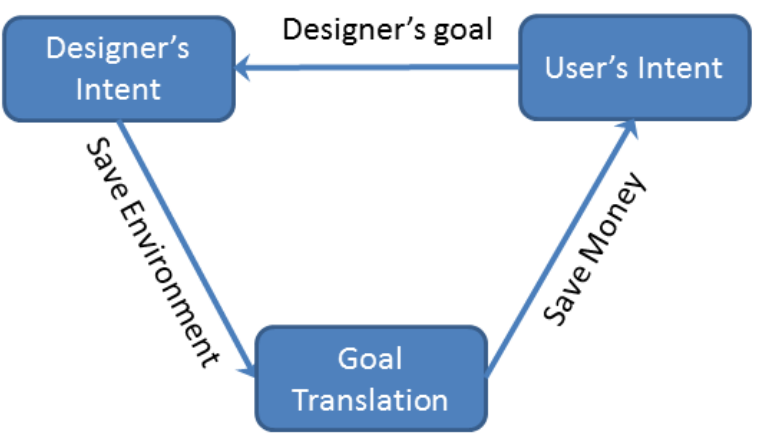

Fig. 1. Translating from Designer Goal to User Goal

raising awareness about the relationship between number of electric units consumed and the resulting saving in money. Here one must consider the fact that this will change the design of the application significantly and introduce some sort of model to take care of money saving but it will also result in a thought process of doing the translation. Such translation may be done easily for both mathematical and persuasion point of view or it may require detailed knowledge of the domain (e.g., environmental factors) by the designer. This concept is explained in 2 .

However, not all users may have the same intent and this strategy may not be helpful in changing their motivation or attitude for electricity usage. The reason could be cheap electricity tariffs or the lack of interest by the user in saving money. To solve such differences, one needs to consider different users' different intents, and for that persuasion facets are used.

\section{B. Persuasion Facets}

When building a persuasive system, the designer is mostly focused on achieving one particular goal (of achieving the specific, desired behavior). For effective persuasion, however, multiple facets of the persuasion process are to be considered. For example, for reducing electricity consumption one persuasion facet is to emphasize on saving money. Another facet can be emphasizing usage of alternative, renewable energy means such as solar-energy-based electricity generation and usage. Yet another facet may be to persuade the users to use smart meters that can identify to the users certain appliances whose usage can be tracked and adjusted according to users' need, e.g., heating systems whose thermostats can be optimized for better economy of electricity.

The designer of a persuasive system should identify different possible facets and then apply persuasion according to the selected facet. Each of the persuasion facets has to be mapped to some user intent clearly. Nevertheless, this mapping cannot be done without considering some additional factors: persuasion context and user preferences.

1) Persuasion Context: Given that persuasion has various facets, persuasion context plays an important role to assist the designer in choosing the most appropriate facet according to a given user's intent. In other words, given different facets and user intents, the persuasion context serves as a mapping function from some facet onto some intent. In literature, the term context is defined as any information that can be used to characterize the situation of an entity [19]. Below are given consider some examples of context.

Having understood the various facets and user intents in the persuasion problem of reducing electricity consumption, a designer can identify a number of entities as context. For example, considering current season or weather as context will allow understanding the appropriate user intent that will in turn determine the appropriate persuasion facet. When the weather or season in the context is cold, it will not be appropriate to consider the option of reducing heater consumption as an economy measure. On the other hand, on warm and sunny areas, it is more appropriate to suggest using alternative means such as solar energy. Other persuasion contexts for this example may include location of usage (e.g., home, office, hotel, or other public place) and time (morning, evening, or night). More sophisticated context entities such as user's education, income, family size, age group, habits or routine, etc. may also needed to be considered in advanced cases.

2) User Preferences: Another factor or function that will help a designer in mapping a persuasion facet onto a user intent is to consider user preferences. To understand the mapping function of user preferences, let's assume that some users may never like the idea of investing into or thinking about alternative energy means including solar energy, because of little motivation or ability. Such likings or dislikes of the user must be considered by the designer carefully because user preferences may be a stronger function than the context function. In case of a conflict between the two or even if a tie occurs between them the user preference will override the context function. Thus, even in warm sunny areas, such users may not be persuaded for using alternative energy means.

\section{Feedback is important in Persuasive Design}

How does a designer know which of the facets the user is interested in? One possible way is to ask the user in the beginning. However, this approach is not flexible and also some users may not be sure about their goals. The designer may need to determine this indirectly using user's behavior. For this purpose, a feedback mechanism is involved from the system or application to the user and vice-versa. So initially, the system has no knowledge about the user but as the system learns about the user as part of the user's interaction with the system, the latter provides more and more feedback in that particular aspect. For example, having shown the user all the facets towards meeting the goal, the system may monitor user's interaction with the provided feedback (electricity consumption, meter's reading, etc.) and will adapt the interface showing more detailed related to the facet in which the user is interested bringing more of persuasion in it.

\section{Persuasion has Several Levels}

The author proposes that to achieve a target behavior, a user may sometimes need to go through several levels (or cells in the behavior grid) before arriving at the final level of the target behavior (unless the behavior is so easy to do or obvious for the user that he take a direct start in the final level). This is where the notion of levels is defined in persuasion similar to levels in computer or video games. 


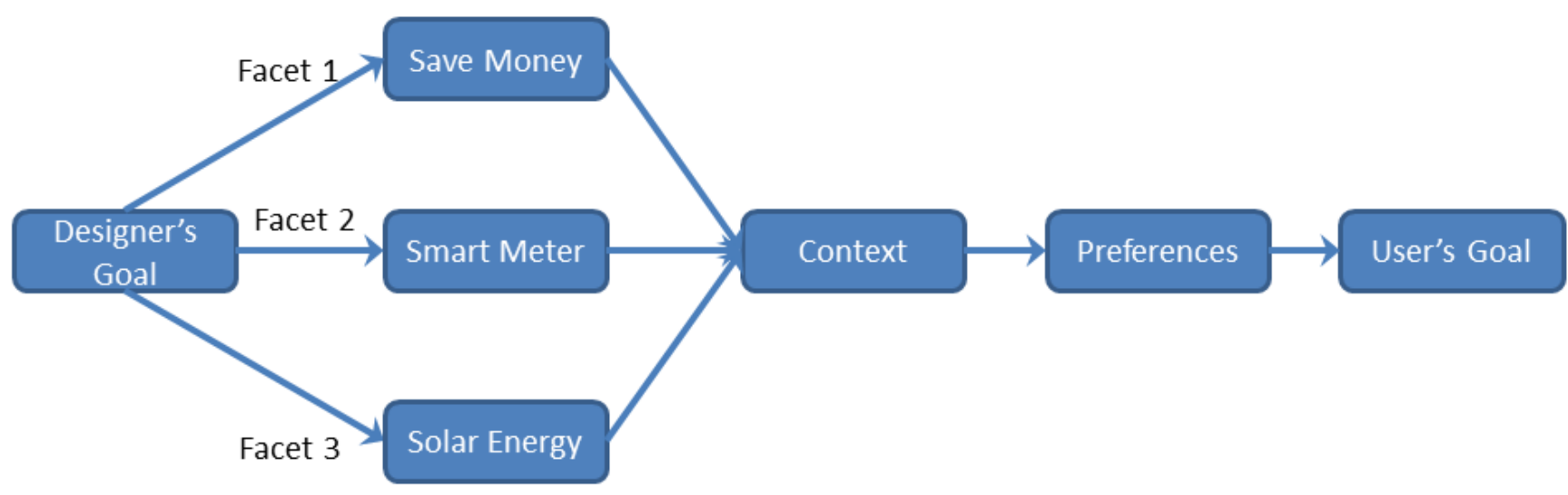

Fig. 2. Persuasion Facets as Alternative Translations into User Goals

Depending upon the persuader's goal, user's goal, persuasion facets and translation functions, it is the job of the persuasion designer to identify the levels required in achieving the target behavior. Although in the current work does not consider any mechanisms on how a designer could do this task, it can be said safely that one possible approach will be to consider the Fogg's notion of tiny habits ${ }^{1}$. Stated simply, the designer may start with very easy to do behaviors that can be converted into habits with least of efforts. As the user establishes himself in a given level (or cell), he may be moved on to the next level with relatively difficult target behavior to do. This may continue from level to level until the desired target behavior is achieved in the final level.

For the given example, the designer may initially persuade the user to install solar energy panel for one room or device: a one-time behavior. Once this is achieved, the user may be persuaded to install the panels for the whole house or additional rooms. This will be successful only if the user sees the benefit of the single installation which can be done by providing feedback to the user on comparative saving of the two energy-consuming mechanisms.

\section{E. Persuasion is a Process}

Instead of devising one strategy, the designer may need to identify a number of levels that will be carried out by user as a "process" before doing the desired target behavior. This may apply to even the act of performing a new behavior onetime only. For instance, in the current example of reducing electricity consumption, the most useful behavior would be to use alternate energy means. This designer goal can be achieved efficiently if the user performs a one-time behavior of investing in the solar energy panels. However, to do that one time behavior, which is quite difficult for the user in terms of motivation, the user may first need to go through other persuasion levels that will increase the motivation of the user sufficient enough that a simple trigger may lead him to perform the behavior of investing in alternative means. This process has been depicted in the model described next.

\footnotetext{
${ }^{1}$ http://tinyhabits.com
}

\section{Towards Analytical Model for Persuasion}

In the previous section, various issues were discussed that need to be addressed in order to introduce analytics in the persuasive design process. In this section, building on the previous concepts, it is explained as how they can be useful in the development of appropriate analytical and mathematical models.

\section{A. The Core Model}

Figure 3 shows a model for an analytical persuasive system after considering these factors. The important thing to note is that this model is cyclic by nature. It has been modeled as a continuous process consisting of some iterations. During each iteration the user's behavior is observed or monitored through his actions or activities while considering the context and user preferences. At the end of the iteration, an assessment is made about the user's actual behavior. While monitoring for the behavior, an analysis is performed in tandem and based upon changes in the context, the most appropriate persuasion facet is applied.

Note that the model contains two cycles. The micro-cycle is used to choose an appropriate facet while the macro-cycle allows the persuader to intervene and make an informed assessment to change the persuasion level. However, this intervention may not be required or desirable in most cases and many systems may do well without this.

Such models must define some way of tracking or measuring the user behavior so that it can be identified in which persuasion level to place the user at a certain time. In addition, they should facilitate us in choosing the appropriate persuasion facet by provide insight into the context and user preferences functions. This is where the importance of user profiling comes into play.

\section{B. User Profiling}

Data plays an important role in analytics and no analytics are possible without having relevant data. That is why the core model contains a profiling component which serves as initial data gathering mechanism for identifying user preferences. However, the process of data gathering continues in each 


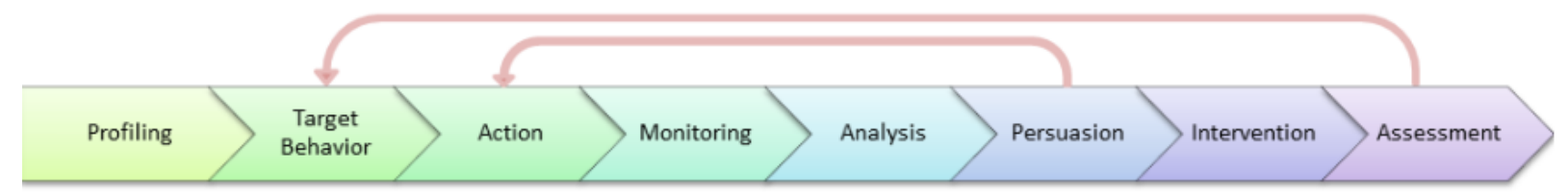

Fig. 3. A model for analytical persuasion

iteration in the form of behavior monitoring. Without data gathering, one will not be able to infer context and choose the appropriate persuasion facet. Such data logging techniques are at the heart of various ubiquitous systems [2][3] and have been used for persuasion [7] as well. It is through the process of user profiling that the designer can infer about user's goal to help them in choosing the appropriate persuasion facet.

\section{User Modeling}

To selectively log data that can be used for persuasion, the user's actions must be understood. This requires the development of appropriate user models specific to each problem. Developing a user model is challenging task as it requires considering some detailed analysis of user's explicit or implicit actions [20][21]. This user model will be used for defining the profiling stage of the core model as well as for specifying what to monitor in each iteration. Most of the times, such models will be a computer representation of the user alongside some mathematical equations to allow reasoning on the data [22][23].

\section{Case Study: Sedentaware}

In this section, the author explains how analytical modeling can be applied for persuasion in some previous work [10]. Sedentaware is mobile application to raise awareness about sedentary behavior in users and to persuade them to do physical activities, if and only when needed. While designing the application, we had to consider several analytical issues as identified above and briefly described here.

Sedentaware uses appropriate alarms (or triggers) to motivate users to exercise. However, unlike many of the similar approaches developed previously, the app uses a mathematical model to determine the appropriate moment for alarm. This concept is depicted in figure 4, adapted from our previous work [10] for four different users with varying levels of behavior. As it can be observed, user 1 is active $50 \%$ of the time and gets no alarm (or trigger), because he does not need one. As the sedentary behavior of the users increase, they get more and more alarms. In other words, based on the mathematical model the persuasion is adaptive to individual user.

Moreover, a number of persuasion facets were identified. Figure 5 shows four different strategies with the intention of motivating the users to participate more in active behavior. First, in figure 5(a) provides a glimpse of user's current progress. A user is motivated if he sees that he is lacking in progress at a given moment. Second, figure 5(b) provides a daily statistics view as a graph of percentages allowing the user to reflect on his progress over some time. Third, figure 5(c) provides a weekly progress view as a different graph as minutes of different activities performed. This graph provides another facet or window to view their behavior differently.
Finally, a fourth persuasion facet is provided in the form of instinct cues to the user. The figure shows an icon on the top left of the mobile screen whose color provides the user a cue on how well he is doing. In this example, these facets are just looking at the same view from different windows. However, the same mathematical model can be used to introduce ranking of users in his social network resulting in a completely different facet of the persuasion strategy of 'competition' with the peers.

Behind these different persuasion facets exist the various elements of a mathematical model that computes the ratio of user's active behavior (walking, running, etc.) to the sedentary behavior. The behavior is detected using activity recognition on user's mobile phone. Interested readers are referred to [10] for further details on development of the model, the data logging procedure used in the application as well as the evaluation by test users. Note that the application translates the persuader goal of prevention of sedentary behavior into the activeness goal of the user. The context of the user (location and time) is also considered. Finally, as the user is adopting the desired behavior, he/she is also progressing in the levels as shown in figure 6 .

As shown in figures 4-6, this model is not only used in designing various persuasion facets but also for evaluating the user's performance by them or even by the designer, assuming the designer has access to the data using appropriate means.

\section{CONCLUSIONS}

In this article, we described the use of analytics in the field of persuasive technology for better design choices to the persuasive system designers. We described several factors to consider when designing for analytics in persuasion and proposed a model that considered persuasion as a process. Using a mobile application, called Sedentaware, as an example for preventing sedentary behavior by motivating users to carry out physical activities, we explained how our model can be used alongside a mathematical model to create enhanced persuasive technologies. The current work identified an important limitation of persuasive technology theory that it lacks the detail required by the designers to carry out systematic process of designing persuasive technologies.

This work will be extended to include further concepts from the persuasion and behavior theory, particularly the work related to Behavior Change Support Systems (BCSS). We also need to identify how different persuasion intents can be mapped to Fogg's behavior grid. It will only be at that time when we will be confident about enhanced role of researchers from the field of computer sciences and relevant domains in persuasive technologies. 


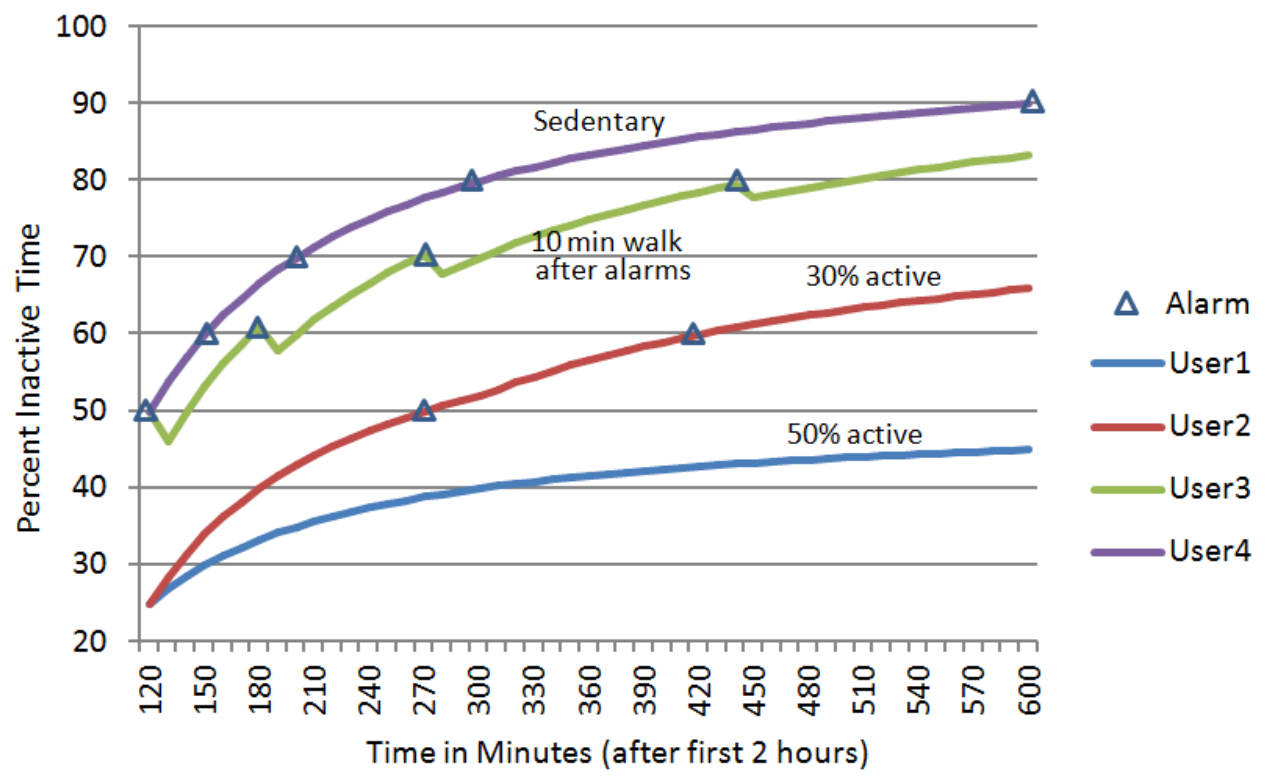

Fig. 4. Results of simulation showing sending of adaptive reminders to various users with different sedentary behavior. Active users receive fewer and less frequent reminders as compared to sedentary users.

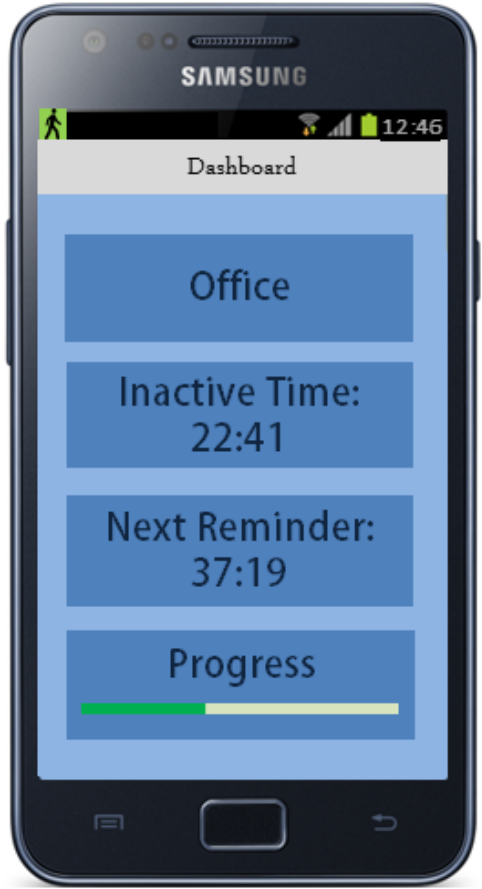

a)

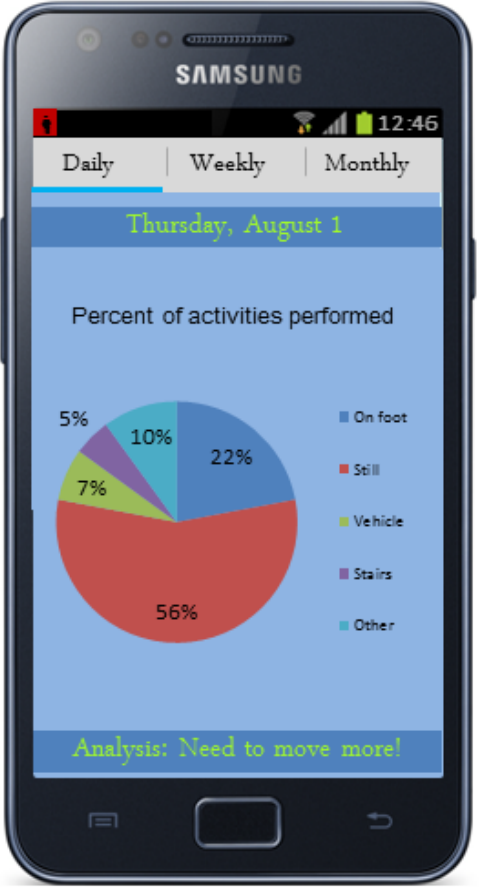

b)

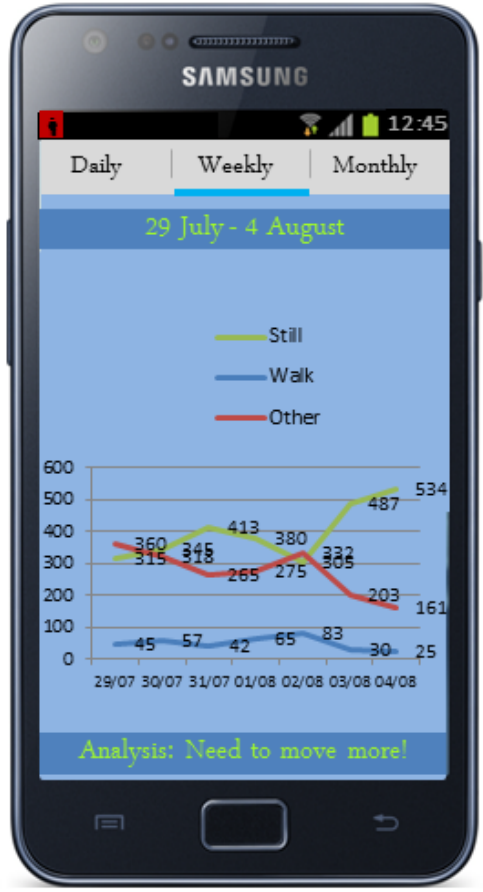

c)

Fig. 5. a) Activity Dashboard b) Summary of Daily Activities c) Weekly Summary of Activities. Both the notification bar and the application's screen reflect the change using various colors 


\section{SmartFit}
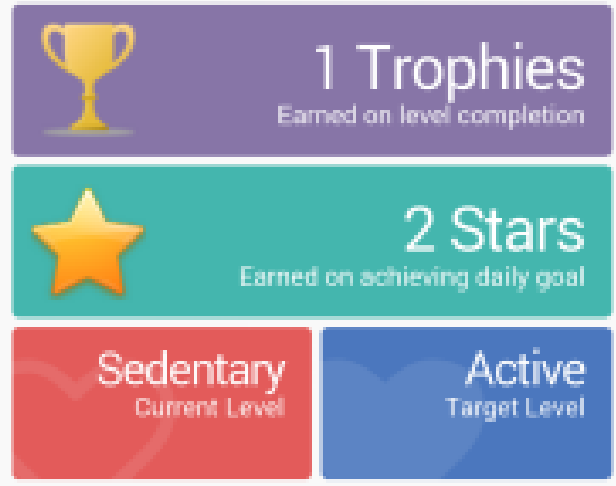

3 more Levels to Go

Sederdary, Lightly Active, Mloderalely Actine

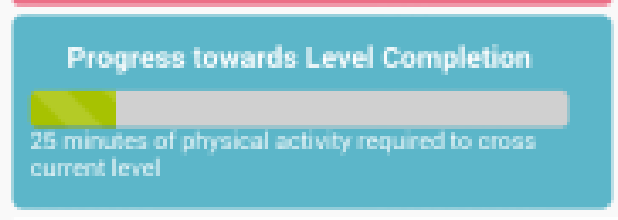

Fig. 6. Feedback and Levels
[12] H. Mukhtar, A. Ali, D. Belaïd, and S. Lee, "Persuasive healthcare selfmanagement in intelligent environments," in Intelligent Environments (IE), 2012 8th International Conference on. IEEE, 2012, pp. 190197.

[13] H. Oinas-Kukkonen, "A foundation for the study of behavior change support systems," Personal and Ubiquitous Computing, pp. 1-13, 2012.

[14] _ - "Behavior change support systems: A research model and agenda," in Persuasive Technology. Springer, 2010, pp. 4-14.

[15] S. Consolvo, J. Landay, and D. McDonald, "Designing for behavior change in everyday life," IEEE Computer, vol. 405, pp. 100-103, 2009.

[16] B. Fogg, "The behavior grid: 35 ways behavior can change," in Proceedings of the 4th international Conference on Persuasive Technology. ACM, 2009, p. 42.

[17] B. Fogg and J. Hreha, "Behavior wizard: a method for matching target behaviors with solutions," Persuasive Technology, pp. 117-131, 2010.

[18] I. Li, A. K. Dey, and J. Forlizzi, "Using context to reveal factors that affect physical activity," ACM Transactions on Computer-Human Interaction (TOCHI), vol. 19, no. 1, p. 7, 2012.

[19] A. K. Dey, "Understanding and using context," Personal and ubiquitous computing, vol. 5, no. 1, pp. 4-7, 2001.

[20] S. McBurney, N. Taylor, H. Williams, and E. Papadopoulou, "Giving the user explicit control over implicit personalisation," in Procs. of Workshop on Intelligent Pervasive Environments (under AISB09), Edinburgh, Scotland, 2009.

[21] Z. Jrad, M.-A. Aufaure, and M. Hadjouni, "A contextual user model for web personalization," in Web Information Systems Engineering-WISE 2007 Workshops. Springer, 2007, pp. 350-361.

[22] J. Fink and A. Kobsa, "User modeling for personalized city tours," Artificial intelligence review, vol. 18, no. 1, pp. 33-74, 2002.

[23] D. Heckmann, Ubiquitous user modeling. IOS Press, 2005, vol. 297.

\section{REFERENCES}

[1] B. Fogg, "Persuasive technology: using computers to change what we think and do," Ubiquity, vol. 2002, no. December, p. 5, 2002.

[2] H. Kimura, J. Ebisui, Y. Funabashi, A. Yoshii, and T. Nakajima, "idetective: a persuasive application to motivate healthier behavior using smart phone," in Proceedings of the 2011 ACM Symposium on Applied Computing, ser. SAC '11, 2011, pp. 399-404.

[3] S. van Dantzig, G. Geleijnse, and A. T. van Halteren, "Toward a persuasive mobile application to reduce sedentary behavior," Personal and Ubiquitous Computing, pp. 1-10, 2011.

[4] B. Fogg, "Creating persuasive technologies : An eight-step design process," in Persuasive '09, 2009, p. 16.

[5] K. Torning and H. Oinas-Kukkonen, "Persuasive system design: state of the art and future directions," in Proceedings of the 4th International Conference on Persuasive Technology. ACM, 2009, p. 30.

[6] T. Alahäivälä, H. Oinas-Kukkonen, and T. Jokelainen, "Software architecture design for health bcss: case onnikka," in Persuasive Technology. Springer, 2013, pp. 3-14.

[7] S. M. Kelders and J. E. L. van Gemert-Pijnen, "Using log-data as a starting point to make ehealth more persuasive," in Persuasive Technology. Springer, 2013, pp. 99-109.

[8] D. Pavel, V. Callaghan, and A. K. Dey, "Looking back in wonder: How self-monitoring technologies can help us better understand ourselves," in Intelligent Environments (IE), 2010 Sixth International Conference on. IEEE, 2010, pp. 289-294.

[9] B. Fogg, "A behavior model for persuasive design," in Proceedings of the 4th international Conference on Persuasive Technology. ACM, 2009, p. 40.

[10] H. Mukhtar and D. Belaïd, "Using adaptive feedback for promoting awareness about physical activeness in adults," in Accepted for publication in 10th IEEE International Conference on Ubiquitous and Intelligent Computing (UIC) 2013, 2013.

[11] H. Mukhtar, A. Ali, S. Lee, and D. Belaïd, "Personalized healthcare self-management using social persuasion," Impact Analysis of Solutions for Chronic Disease Prevention and Management, pp. 66-73, 2012. 